REVISTA INTERNACIONAL DE CIENCIAS DEL DEPORTE International Journal of Sport Science

Rev. int. cienc. deporte

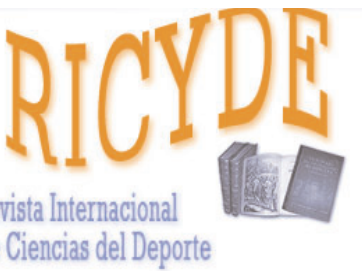

International Journal of Sport Science VOLUMEN X - AÑO X

Páginas:336-345 ISSN:1885-3137

No 38 - Octubre - 2014

\title{
Cambios en la condición física de árbitros de fútbol: un estudio longitudinal Changes in the physical fitness of soccer referees: a longitudinal study
}

\author{
Javier Yanci-Irigoyen \\ Facultad de Ciencias de la Actividad Física y del Deporte, Universidad del País Vasco, UPV/EHU
}

\begin{abstract}
Resumen
El objetivo de este estudio fue conocer la evolución en la condición física de árbitros de fútbol medida a través de la capacidad cardiovascular, la capacidad de salto vertical y la capacidad de aceleración durante tres temporadas distintas. El estudio longitudinal se realizó durante las temporadas 2008$2009,2009-2010$ y 2010-2011. La muestra final seleccionada para participar en este estudio fue de 16 árbitros (edad $=28,37 \pm 5,3$ años, estatura $=1,77 \pm 0,23 \mathrm{~m}$, masa $=74,48 \pm 8,18 \mathrm{~kg}$, grasa corporal $=13,37 \pm 3,72 \%$ ) pertenecientes a distintas categorías del fútbol español. No se encontraron diferencias significativas en el consumo máximo de oxígeno estimado $\left(\mathrm{VO}_{2} \mathrm{maxE}\right)$ ni en el tiempo de agotamiento (TAGOT) en el test de resistencia a lo largo de las tres temporadas en los árbitros de fútbol. Sin embargo, la capacidad de aceleración tanto en $5 \mathrm{~m}$ como en $15 \mathrm{~m}$ empeoró significativamente $(p<0,01, T E>0,8)$ a lo largo de las distintas temporadas. De la misma forma, la capacidad del ciclo estiramiento-acortamiento muscular de las extremidades inferiores mostró una tendencia a empeorar ( $T E=0,53-0,76)$ con el paso del tiempo. La perdida de rendimiento en la capacidad de aceleración y en la capacidad del ciclo estiramiento-acortamiento muscular de las extremidades inferiores nos puede llevar a pensar en la necesidad de implementar programas específicos para su mejora en árbitros de fútbol.
\end{abstract}

Palabras clave: aceleración; sprint; salto vertical; resistencia; rendimiento.

\begin{abstract}
The aim of this study was to determine the evolution of the physical fitness of soccer referees measured by cardiovascular capacity, vertical jumping ability and acceleration capacity for three different seasons. The longitudinal study was conducted over the 2008-2009, 2009-2010 and 2010-2011 seasons. The final sample selected to participate in this study was 16 referees (age $=28.37 \pm 5.3$ years, height $=1.77 \pm 0.23 \mathrm{~m}$, mass $=74.48 \pm 8.18 \mathrm{~kg}$, body fat $=13.37 \pm 3.72 \%$ ) belonging to different categories of Spanish soccer league. No significant differences in the estimated $\mathrm{VO}_{2} \mathrm{max}$ or time to exhaustion (TAGOT) in the endurance test over the three seasons were found in soccer referee. However, the ability to accelerate both $5 \mathrm{~m}$ and $15 \mathrm{~m}$ significantly worse $(\mathrm{p}<0.01, \mathrm{ES}>0.8)$ along the different seasons. Likewise, the ability of the muscle stretch-shortening cycle of the lower extremities showed a worsening trend over time ( $E S=0.53$ to 0.76 ). The loss of performance acceleration capacity and muscle stretch-shortening cycle of the lower extremities capacity can lead us to think about the need to implement specific training programs for improving soccer referees.
\end{abstract}

Key words: acceleration; sprint; vertical jump; endurance; performance.

Correspondencia/correspondence: Javier Yanci Irigoyen

Facultad de Ciencias de la Actividad Física y del Deporte, Universidad del País Vasco, UPV/EHU

Lasarte s/n, 01007 Vitoria-Gastéiz, España

E-mail: javier.yanci@ehu.es 


\section{Introducción}

$\mathrm{L}$ os árbitros de fútbol, junto con los árbitros asistentes son los encargados de supervisar la aplicación de las reglas de juego en los partidos. Se ha estimado que cada semana, aproximadamente 1,3 millones de árbitros en todo el mundo participan en partidos de fútbol con la intención de regular y controlar el cumplimiento de las reglas de juego (Castagna, Abt, y D'Ottavio, 2007). Además, las múltiples decisiones que toman los árbitros en un encuentro, tienen gran implicación en el desarrollo del mismo (Castagna y col., 2007). Sin embargo, dada la importancia de este colectivo en el correcto desarrollo deportivo, son necesarios más estudios científicos de campo donde se valore la condición física en árbitros de fútbol.

Desde el punto de vista de las exigencias físicas, durante un encuentro oficial, un árbitro de fútbol recorre entre 9 y $13 \mathrm{~km}$ aproximadamente al 85-90\% de la frecuencia cardiaca máxima (FCmax) y al 70-80\% del consumo máximo de oxigeno $\left(\mathrm{VO}_{2} \max \right)$ (Castagna y col., 2007; Weston, Castagna, Impellizzeri, Bizzini, Williams, y Gregson, 2012). Un estudio realizado por Krustrup y Bangsbo (2001) observó que los árbitros durante un partido además de requerir un alto nivel de producción aeróbica también presentaban una considerable producción de energía anaeróbica. De esta forma, los árbitros de fútbol realizan una importante cantidad de acciones cortas y de alta intensidad como acelerar, frenar o cambiar de dirección para atender las exigencias del juego (Castagna y col., 2007), por lo que resulta importante que estos deportistas tengan altos niveles de fuerza y capacidad de aceleración. Concretamente las acciones de aceleración suelen tener una duración de 2-4 s y raramente se recorren más de $30 \mathrm{~m}$ seguidos a alta intensidad (D’Ottavio y Castagna, 2001). Por lo tanto, la preparación física de los árbitros cobra especial importancia y debe inducir a adaptaciones fisiológicas que favorezcan los requerimientos necesarios en el juego.

Uno de los parámetros ampliamente analizados en el rendimiento deportivo es la capacidad de salto vertical. Varios estudios recientes han determinado que en deportes de equipo, la comparación o diferencia entre el rendimiento en el salto vertical sin contramovimiento (SJ) y en el salto vertical con contramovimiento $(\mathrm{CMJ})$ es un indicador de la eficiencia del ciclo estiramiento acortamiento (CEA) del tren inferior (Castagna y Castellini, 2013; McGuigan, Doyle, Newton, Edwards, Nimphius, y Newton, 2006). El estudio del CEA en deportistas se ha realizado asiduamente mediante el cálculo del índice elástico (IE) (Cámara, Grande, Mejuto, Los Arcos, Yanci, 2013; González-Montesinos, Caraballo-Vidal, Gómez-Espinosa de los Monteros, Fernández-Santos, y Román-Bazán, 2010) o a través del cálculo de la diferencia de rendimiento en el SJ y en el CMJ (Castagna y Castellini, 2013). A pesar de la importancia que tiene este aspecto en el rendimiento deportivo en deportes de equipo (Bobbert y Casius, 2005), no hemos encontrado ningún estudio que analice el comportamiento del CEA en árbitros de fútbol.

La mayoría de los estudios publicados en la literatura científica se centran en el análisis de los requerimientos físicos y fisiológicos durante los partidos (D’Ottavio y Castagna, 2001; Mallo, Navarro, García-Aranda, y Helsen, 2009; Weston, Castagna, Impellizzeri, Rampinini, y Breivik, 2010; Weston, Drust, y Gregson, 2011) o la evaluación de distintos parámetros para determinar la condición física de los árbitros en condiciones de laboratorio (Casajús y Castagna, 2007; Krustrup y Bangsbo, 2001; Mendiguchia, Los Arcos, Garrues, Myer, Yanci, e Idoate, 2013) o a través de test de campo (Casajús y Castagna, 2007; Castagna, Abt, y D'Ottavio, 2005). Sin embargo, existen pocos estudios donde se analice la evolución de la condición física de los árbitros a lo largo de distintas temporadas. Un estudio de caso realizado por Weston, Gregson, Castagna, Breivik, Impellizzeri, y Lovell (2011) analiza los 
hábitos de entrenamiento, las exigencias en los partidos y las variables fisiológicas en laboratorio durante un periodo de 8 años en un solo árbitro de la liga de fútbol inglesa. Los datos presentados en este estudio proporcionan una visión completa del impacto a largo plazo de un entorno de formación en el rendimiento físico de un árbitro. Sin embargo, son necesarios más estudios al respecto ya que puede resultar interesante conocer los cambios que se producen en la condición física de los árbitros de fútbol a lo largo de las distintas temporadas.

Por lo tanto, el objetivo de este estudio fue conocer la evolución en la condición física de árbitros de fútbol medida a través de la capacidad cardiovascular, la capacidad de salto vertical y la capacidad de aceleración durante tres temporadas distintas.

\section{Método}

\section{Participantes}

En este estudio participaron 57 árbitros pertenecientes al Comité Navarro de Árbitros de Fútbol (CNAF) que militaban en alguna de las categorías tanto nacionales $\left(1^{\mathrm{a}}, 2^{\mathrm{a}}, 2^{\mathrm{a}} \mathrm{B}\right.$ o $3^{\mathrm{a}}$ división) como autonómicas (preferente o regional) de la liga española. Al tratarse de un estudio longitudinal llevado a cabo durante 3 temporadas distintas, la muestra final seleccionada de este estudio no probabilístico fue de 16 árbitros (edad $=28,37 \pm 5,3$ años, estatura $=1,77 \pm 0,23 \mathrm{~m}$, masa $=74,48 \pm 8,18 \mathrm{~kg}$, grasa corporal $=13,37 \pm 3,72 \%$ ), concretamente aquellos que completaron todo el proceso de investigación. Un total de 41 árbitros fueron excluidos del estudio debido a no realizar todos los test en las tres temporadas, por causas de lesión, abandono del arbitraje u otros motivos. Ninguno de los participantes seleccionados realizaba entrenamiento específico de fuerza y todos entrenaban una media de 2-4 sesiones semanales y arbitraban uno o dos partidos cada semana durante la temporada. Todos los participantes tenían una experiencia superior a 8 años en el arbitraje. El estudio se realizó bajo el consentimiento del CNAF. Todos los participantes fueron informados de los objetivos de la investigación, participaron voluntariamente en el estudio, pudieron retirarse del mismo en cualquier momento y firmaron el preceptivo consentimiento informado. Los procedimientos siguieron las pautas marcadas por la Declaración de Helsinki (2013).

\section{Procedimiento}

El estudio longitudinal se realizó durante tres temporadas distintas, la temporada 2008-2009 (T1), la temporada 2009-2010 (T2) y la temporada 2010-2011 (T3). Todos los participantes realizaron dos sesiones de test en cada una de las temporadas, concretamente la segunda semana del mes de octubre, una vez había comenzado la temporada. En la primera sesión, se realizaron las mediciones antropométricas y la prueba de capacidad cardiovascular. En la segunda sesión de test, $48 \mathrm{~h}$ después, se realizaron los test correspondientes a la aceleración en línea recta y al salto vertical. Los test se realizaron en una pista de atletismo con superficie de tartán, lugar donde habitualmente entrenaban los árbitros. Todos los participantes estaban familiarizados con la correcta ejecución de las pruebas, ya que eran los test utilizados habitualmente en las temporadas anteriores. Antes de cada sesión de test, todos los jugadores realizaron un calentamiento que consistió en 5 min de carrera suave, ejercicios de frecuencia de zancada, aceleraciones y saltos verticales.

Test de resistencia: Para determinar la capacidad cardiovascular de los árbitros se utilizó el test de campo de la Universidad de Montreal (UM-TT) (Léger, 1980). El test de la UM-TT se llevó a cabo en una pista de atletismo al aire libre de $400 \mathrm{~m}$, señalizada con conos cada $50 \mathrm{~m}$. La velocidad de carrera se controló a través de una señal sonora pre programada, estableciéndose una velocidad inicial a $6 \mathrm{~km} \cdot \mathrm{h}^{-1}$ que aumentaba $1,20 \mathrm{~km} \cdot \mathrm{h}^{-1}$ cada $2 \mathrm{~min}$. La 
finalización del test para cada jugador se consideró cuando no llegaban por segunda vez a la línea correspondiente en el tiempo marcado (evaluación objetiva) o cuando el propio participante consideraba que no podía continuar en la prueba (evaluación subjetiva) (Castagna, Impellizzeri, Rampinini, D'Ottavio, y Manzi, 2008), midiéndose el tiempo total de duración de la prueba o tiempo de agotamiento (TAGOT) para cada jugador. El $\mathrm{VO}_{2}$ max fue estimado $\left(\mathrm{VO}_{2} \mathrm{maxE}\right)$ a partir del TAGOT, atendiendo a la fórmula propuesta anteriormente por Léger (1980).

Test de salto vertical: Los deportistas realizaron cada uno 3 saltos SJ y otros 3 saltos CMJ atendiendo a los procedimientos marcados por Bosco, Komi, Tihanyi, Fekete, y Apor (1983). El material utilizado para el registro de los resultados fue el Opto Jump Next巴 (Microgate, Italy). En todos los saltos las manos debían permanecer en las caderas durante toda la fase de ejecución (Cámara y col., 2013). Para el SJ, se requirió una flexión de rodillas de $90^{\circ}$ (Bosco y col., 1983). Para los saltos CMJ el ángulo de flexión fue libre y se pidió a los deportistas que realizaran un movimiento natural. Todos los saltos que no cumplían con los requerimientos estipulados fueron excluidos. Se utilizó el tiempo de vuelo para calcular el cambio en la altura del centro de gravedad (Bosco y Komi, 1978; Markovic, Dizdar, Jukic, y Cardinale, 2004). Con el fin de analizar la eficiencia del CEA (Castagna y Castellini, 2013; Maulder y Cronin, 2005) se calculó el índice elástico (IE) atendiendo a la fórmula utilizada anteriormente con jugadores de fútbol: IE $=[(\mathrm{CMJ}-\mathrm{SJ}) \times 100] / \mathrm{SJ}$, donde el IE se mide en $\%$ (González-Montesinos y col., 2010).

Test de aceleración en línea recta: El test de sprint consistió en realizar 3 aceleraciones máximas de $15 \mathrm{~m}$ (Yanci y Los Arcos, 2013) en una pista de atletismo, con un descanso de $180 \mathrm{~s}$ entre cada repetición. Para el registro del tiempo empleado se utilizaron 3 fotocélulas (Powertimer, Newtest ${ }^{\circledR}$ Oy, Oulu, Finland), colocadas en el punto 0, a los $5 \mathrm{~m}$ y a los $15 \mathrm{~m}$. Los deportistas salían desde una línea colocada $50 \mathrm{~cm}$ antes de la primera fotocélula o punto 0 (Yanci y Los Arcos, 2013).

\section{Análisis estadístico de los datos}

Los resultados se presentan como media \pm desviación típica de la media. Todas las variables mostraron una distribución normal según el test de Kolmogorov-Smirnov. Para el análisis de los resultados en los test de aceleración y de salto, se consideró únicamente el mejor de los tres registros. Las diferencias de los distintos parámetros analizados a lo largo de las tres temporadas se calcularon mediante un ANOVA de medidas repetidas, junto con el correspondiente análisis pos hoc de Bonferroni. Para determinar las diferencias existentes entre los resultados en el SJ y en el CMJ se utilizó una prueba T para muestras relacionadas. El tamaño del efecto (TE) se calculó atendiendo al método propuesto por Cohen (1988). Tamaños del efecto menores a 0,2 , entre $0,2-0,5$, entre $0,5-0,8$ o mayores de 0,8 fueron considerados trivial, bajo, moderado o alto, respectivamente. El análisis estadístico se realizó con el programa Statistical Package for Social Sciences (SPSS Inc, versión 20,0 Chicago, IL, EE.UU.). La significatividad estadística fue de $p<0,05$.

\section{Resultados}

\section{Capacidad cardiovascular}

En este estudio no se encontraron diferencias significativas en el $\mathrm{VO}_{2} \operatorname{maxE}(\mathrm{F}(2)=1,00, \mathrm{p}>$ $0,05)$ ni en el TAGOT $(\mathrm{F}(2)=0,73, \mathrm{p}>0,05)$ en el test de la UM-TT a lo largo de las tres temporadas en los árbitros de fútbol (Tabla 1). El tamaño del efecto para el $\mathrm{VO}_{2} \mathrm{maxE}$ fue trivial en $\mathrm{T} 1-\mathrm{T} 2(\mathrm{TE}=0,12)$ y en $\mathrm{T} 1-\mathrm{T} 3(\mathrm{TE}=0,04)$. Sin embargo se obtuvo un tamaño del efecto moderado $(\mathrm{TE}=0,50)$ entre $\mathrm{T} 2$ y $\mathrm{T} 3$. Resultados similares se obtuvieron con respecto 
al TAGOT en la prueba donde no se encontraron diferencias significativas entre las distintas temporadas, con tamaños de efecto triviales $(\mathrm{TE}=0,04-0,18)$.

\begin{tabular}{|c|c|c|c|}
\hline & T1 & $\mathrm{T} 2$ & T3 \\
\hline $\mathrm{VO}_{2}$ max estimado $\left(\mathrm{ml} \cdot \mathrm{kg}^{-1} \cdot \mathrm{min}^{-1}\right)$ & $56,47 \pm 3,99$ & $56,00 \pm 2,71$ & $56,64 \pm 4,65$ \\
\hline TAGOT (min) & $20,84 \pm 2,04$ & $20,76 \pm 2,01$ & $21,22 \pm 2,77$ \\
\hline
\end{tabular}

\section{Capacidad de salto vertical}

Los resultados obtenidos en el SJ, CMJ y el IE se muestran en la tabla 2. En todas las temporadas analizadas se obtuvieron diferencias significativas entre el SJ y el CMJ (T1, t(15) $=14,81, \mathrm{p}<0,01 ; \mathrm{T} 2, \mathrm{t}(15)=12,62, \mathrm{p}<0,01 ; \mathrm{T} 3, \mathrm{t}(15)=6,01, \mathrm{p}<0,01)$. No se encontraron diferencias significativas a lo largo de las tres temporadas en la capacidad de salto vertical. Sin embargo, a pesar de que no se obtuvieron diferencias significativas en el IE $(\mathrm{F}(2)=0,431$, $\mathrm{p}>0,05)$, el tamaño del efecto para el IE fue moderado entre la temporada T1-T2 $(\mathrm{TE}=0,53)$ y entre la temporada $\mathrm{T} 1-\mathrm{T} 3(\mathrm{TE}=0,76)$.

Tabla 2. Resultados en el squat jump (SJ), el salto con contra movimiento (CMJ) y el índice elástico (IE) a lo largo de las tres temporadas $(\mathrm{n}=16)$.

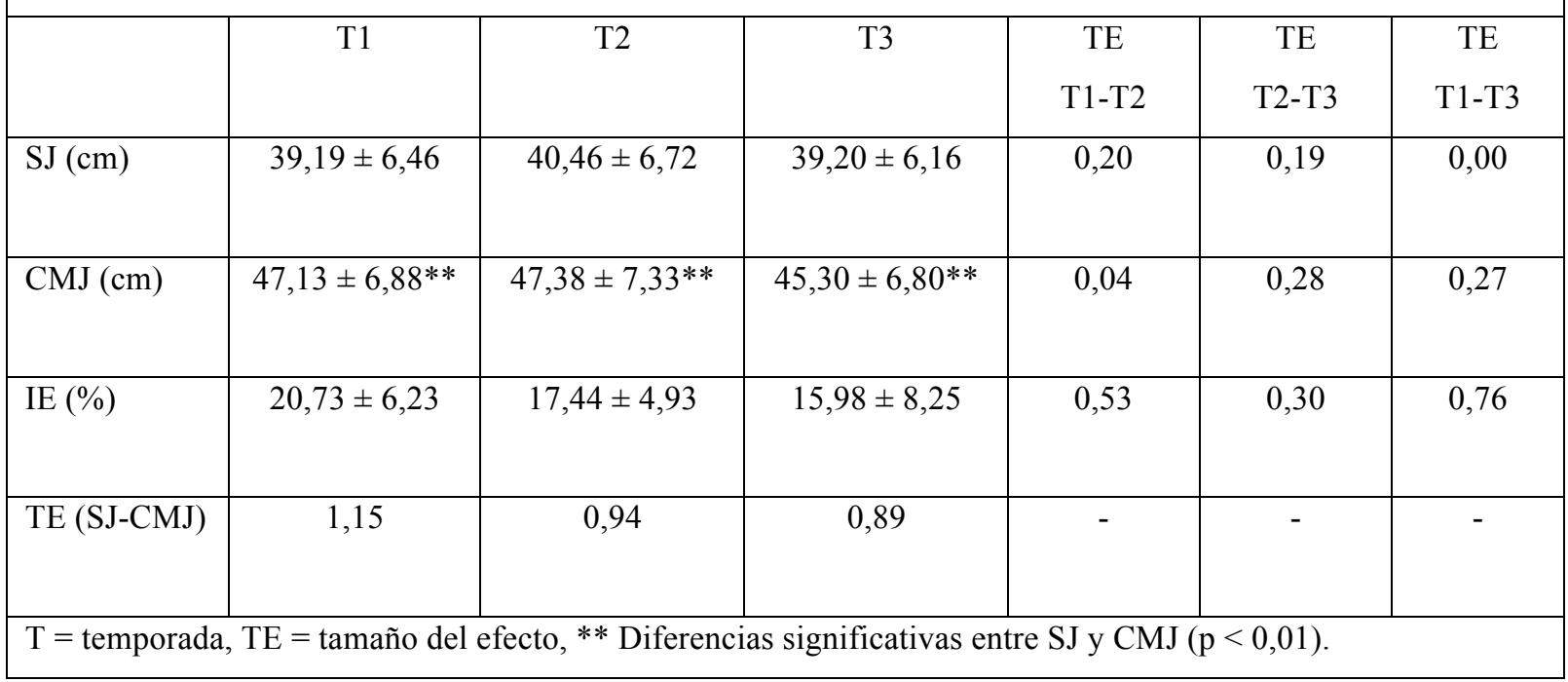

\section{Capacidad de aceleración}

Los resultados de las tres temporadas en cuanto a la capacidad de aceleración en 5 m y $15 \mathrm{~m}$ se muestran en la Figura 1. Se obtuvieron diferencias significativas $(\mathrm{p}<0,01)$ tanto en el tiempo empleado en recorrer los $5 \mathrm{~m}(\mathrm{~F}(2)=29,58, \mathrm{p}<0,01)$ como los $15 \mathrm{~m}(\mathrm{~F}(2)=21,87, \mathrm{p}$ $<0,01$ ) entre las distintas temporadas T1-T2 y T1-T3), con tamaños del efecto altos (TE $>$ $0,8)$. 


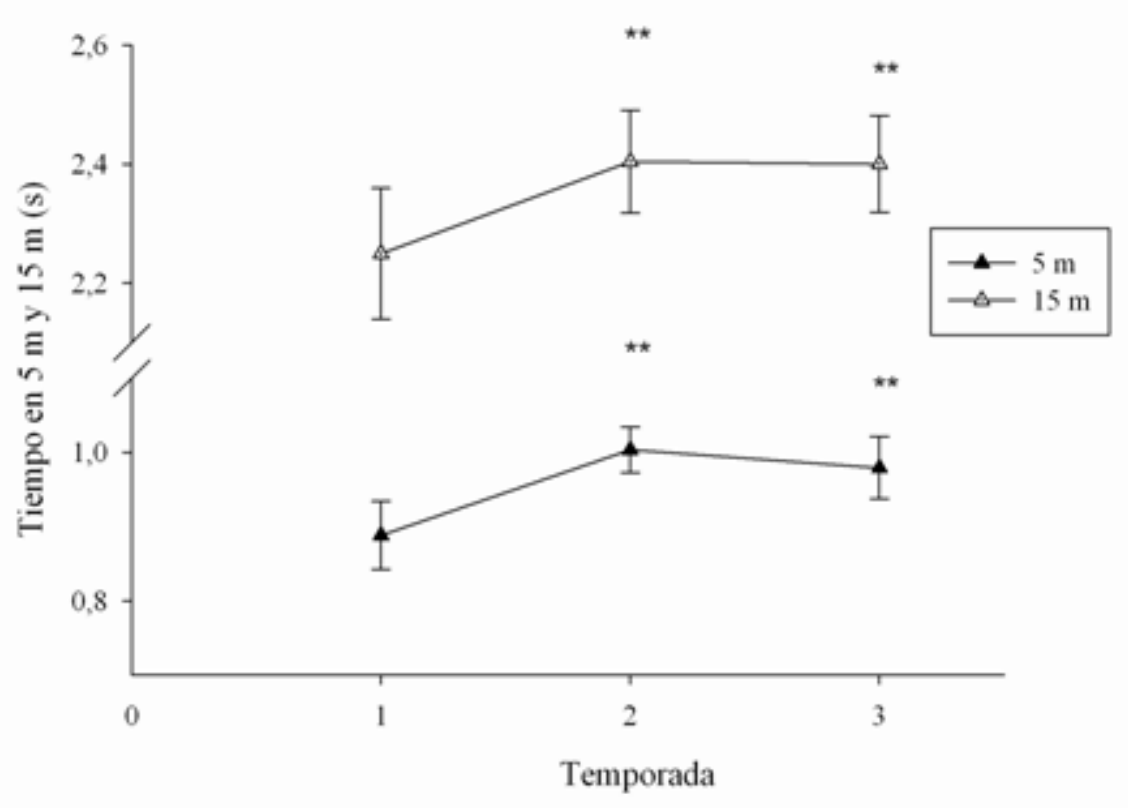

Figura 1. Tiempo en el test de $5 \mathrm{~m}$ y $15 \mathrm{~m}$ durante las tres temporadas.

** Diferencias significativas $(\mathrm{p}<0,01)$ con respecto a la temporada 1 (T1)

\section{Discusión}

El propósito de este estudio fue analizar las variaciones en la condición física de árbitros de fútbol a lo largo de tres temporadas distintas. Para nuestro conocimiento, este es el primer estudio longitudinal donde se evalúan los cambios en el rendimiento cardiovascular, la capacidad de salto vertical y la capacidad de aceleración en árbitros de fútbol en tres temporadas consecutivas. Los principales hallazgos de este estudio fueron que la capacidad de aceleración tanto en el test de $5 \mathrm{~m}$ como en $15 \mathrm{~m}$ empeoró significativamente a lo largo de las distintas temporadas. De la misma forma, la capacidad del ciclo estiramiento-acortamiento muscular de las extremidades inferiores mostró una tendencia a empeorar con el paso del tiempo. Sin embargo, la capacidad cardiovascular de los árbitros mostró una tendencia de mejoría únicamente entre la segunda y tercera temporada pero no entre la primera y tercera temporada.

El rendimiento del metabolismo aeróbico correlaciona fuertemente con el rendimiento físico durante el arbitraje en los partidos de fútbol de distintas categorías y niveles competitivos (Castagna y col., 2007). Además, estudios anteriores determinan que el $\mathrm{VO}_{2} \mathrm{max}$ de los árbitros afecta directamente al desempeño en los partidos (Castagna y D'Ottavio, 2001; Krustrup y Bangsbo, 2001). Atendiendo a los resultados obtenidos en nuestro estudio, y a pesar del paso del tiempo, no se encontraron diferencias significativas en el $\mathrm{VO}_{2} \mathrm{maxE}$ ni en el tiempo de agotamiento en los árbitros de fútbol a lo largo de las tres temporadas. Sin embargo, el tamaño del efecto moderado $(\mathrm{TE}=0,50)$ encontrado entre el T2 y el T3 en cuanto al $\mathrm{VO}_{2}$ max estimado, pone de manifiesto una tendencia en la mejora de esta capacidad. Estudios anteriores realizados con futbolistas a lo largo de una sola temporada muestran valores similares. Magal, Smith, Dyer, y Hoffman (2009) reportan pequeñas mejorías a lo largo de la temporada en jugadores jóvenes. Por su parte, Oliveira, Leicht, Bishop, BarberoÁlvarez, y Nakamura (2013) en un estudio con jugadores de fútbol sala, observaron que los 
índices de rendimiento cardiovascular en un test de campo se mantenían constantes a lo largo de la temporada, si bien mejoraban ligeramente en pretemporada. El hecho de que la capacidad cardiovascular no haya descendido a lo largo de las tres temporadas en nuestro estudio puede deberse, en parte, a que los árbitros participaban una vez por semana en partidos oficiales a lo largo de las temporadas, donde los requerimientos aeróbicos son altos (Castagna y col., 2007). Además, los entrenamientos realizados por los árbitros participantes en el estudio tenían un alto contenido de trabajo de resistencia. Estos dos aspectos han podido influir en la tendencia de mejora de esta cualidad después de tres temporadas. Por lo tanto, parece ser que el estimulo de los partidos junto con entrenamientos genéricos de resistencia, pueden ser suficientes para mantener el rendimiento cardiovascular a lo largo de distintas temporadas.

El rendimiento en el salto vertical durante las diferentes temporadas tampoco sufrió modificaciones significativas. Los resultados tanto en el SJ como en el CMJ de los árbitros se mantuvieron constantes a lo largo de las tres temporadas. Sin embargo, aunque no significativa, los árbitros de fútbol mostraron una pérdida del índice elástico entre las temporadas T1-T2 $(\mathrm{TE}=0,53$, moderada) y T1-T3 $(\mathrm{TE}=0,76$, moderada). La capacidad del ciclo estiramiento-acortamiento se vio influenciada negativamente por el paso del tiempo. Recientemente, el interés, en el análisis deportivo, sobre la potenciación de rendimiento en los movimientos de estiramiento-acortamiento ha crecido (Bobbert y Casius, 2005). La comparación entre la altura en el SJ y en el CMJ había sido evaluada para determinar la eficiencia del CEA en las extremidades inferiores (Castagna y Castellini, 2013; McGuigan y col., 2006). Sin embargo, a pesar de la importancia que puede tener para el rendimiento (Castagna y Castellini, 2013), hasta el momento ningún estudio había analizado este aspecto en árbitros de fútbol. El descenso en el índice elástico de los árbitros, nos puede llevar a pensar en la necesidad de implementar entrenamientos específicos para paliar la perdida de rendimiento del ciclo estiramiento-acortamiento a lo largo de las distintas temporadas.

Durante los partidos oficiales, un árbitro de fútbol puede llegar a recorrer a velocidad de sprint una distancia de hasta el 11,8\% de la distancia total recorrida en el partido (Krustrup, Helsen, Randers, Christensen, MacDonald, Rebelo, y Bangsbo, 2009), por lo que las acciones cortas e intensas pueden tener especial importancia. Los resultados de nuestro estudio muestran un descenso significativo entre T1-T2 y T1-T3 en el rendimiento tanto en $5 \mathrm{~m}$ como en $15 \mathrm{~m}$. Los árbitros de fútbol participantes en este estudio experimentaron una pérdida significativa en la capacidad de aceleración a medida que trascurrían las temporadas. Estudios anteriores han determinado que la capacidad de aceleración y de realizar acciones a alta intensidad permiten a los árbitros estar más cerca del juego, pudiendo realizar así una mejor observación y análisis de las acciones de los futbolistas (Casajús y Castagna, 2007). De esta forma, podría resultar adecuado implementar programas específicos para evitar la pérdida de la capacidad de aceleración en los protocolos de entrenamiento de los árbitros de fútbol.

Una de las principales limitaciones de nuestro estudio ha podido ser la heterogeneidad de la muestra, ya que en el mismo han participado árbitros de distintas categorías y por lo tanto con distintos niveles de rendimiento y entrenamiento. Este aspecto puede haber influido en los resultados obtenidos. Por lo tanto, los resultados hay que tomarlos con cautela y puede resultar interesante realizar más investigaciones al respecto con árbitros de categorías similares y una muestra más homogénea. 


\section{Conclusiones}

Los resultados obtenidos en el presente estudio muestran que la capacidad de aceleración de los árbitros de fútbol fue significativamente diferente en las tres temporadas analizadas. De la misma forma, en el índice elástico de las extremidades inferiores se observo una tendencia a empeorar con el paso del tiempo. La perdida de rendimiento en estas capacidades y su importancia para el correcto desempeño en los partidos de fútbol nos puede llevar a pensar en la necesidad de implementar programas específicos para mejorar tanto la capacidad de aceleración como la capacidad del ciclo estiramiento-acortamiento muscular de las extremidades inferiores.

Sin embargo, la capacidad cardiovascular siguió una tendencia contraria. Parece ser que el estimulo de los partidos junto con entrenamientos genéricos de resistencia, pueden ser suficientes para mantener el rendimiento cardiovascular a lo largo de distintas temporadas.

\section{Agradecimientos}

Queremos agradecer al Comité Navarro de Árbitros de Futbol (CNAF) por ofrecernos la posibilidad de realizar esta investigación y en concreto a los árbitros participantes en este estudio.

\section{Referencias}

Bobbert, M. F., \& Casius, L. J. (2005). Is the effect of a countermovement on jump height due to active state development? Medicine and Science in Sports and Exercise, 37, 440-446.

http://dx.doi.org/10.1249/01.MSS.0000155389.34538.97

Bosco, C., \& Komi, P. V. (1978), Mechanical characteristics and fiber composition of human leg extensor muscles. European Journal of Applied Physiology and Occupational Physiology, 41(4), 275-284.

http://dx.doi.org/10.1007/BF00429744

Bosco, C.; Komi, P. V.; Tihanyi, J.; Fekete, G., \& Apor, P. (1983), Mechanical power test and fiber composition of human leg extensor muscles. European Journal of Applied Physiology and Occupational Physiology, 51(1), 129-135.

http://dx.doi.org/10.1007/BF00952545

Cámara, J.; Grande, I.; Mejuto, G.; Los Arcos, A., \& Yanci, J. (2013). Jump landing characteristics in elite soccer players with cerebral palsy. Biology of Sport, 30(2):91-95. http://dx.doi.org/10.5604/20831862.1044223

Casajús, J.A., \& Castagna, C. (2007). Aerobic fitness and field test performance in elite Spanish soccer referees of different ages. Journal of Science and Medicine in Sport, 10(6), 382-389.

http://dx.doi.org/10.1016/j.jsams.2006.08.004

Castagna, C., \& D'Ottavio, S. (2001). Effect of maximal aerobic power on match performance in elite soccer referees. Journal of Strength and Conditioning Research, 15 (4), 420-425.

Castagna, C.; Abt, G., \& D'Ottavio, S. (2005). Competitive-level differences in Yo-Yo intermittent recovery and twelve minute run test performance in soccer referees. Journal of Strength and Conditioning Research, 19(4), 805-809.

Castagna, C.; Abt, G., \& D'Ottavio, S. (2007). Physiological aspects of soccer refereeing performance and training. Sports Medicine, 37, 625-646.

http://dx.doi.org/10.2165/00007256-200737070-00006 
Castagna, C.; Impellizzeri, F. M.; Rampinini, E.; D'Ottavio, S., \& Manzi, V. (2008). The Yo-Yo intermittent recovery test in basketball players. Journal of Science and Medicine in Sport, 11(2), 202-208.

http://dx.doi.org/10.1016/j.jsams.2007.02.013

Castagna, C., \& Castellini, E. (2013). Vertical jump performance in Italian male and female national team soccer players. Journal of Strength and Conditioning Research, $27(4), 1156-1161$.

http://dx.doi.org/10.1519/JSC.0b013e3182610999

Cohen J. (1988). Statistical power analysis for the behavioral sciences. Hillsdale, NJ: Lawrence Erlbaum Associates.

D'Ottavio, S., \& Castagna, C. (2001). Analysis of match activities in elite soccer referees during actual match play. Journal of Strength and Conditioning Research, 15(2), 167171.

http://dx.doi.org/10.1519/1533-4287(2001)015<0167:AOMAIE > 2.0.CO;2

González-Montesinos, J.L.; Caraballo-Vidal, I.; Gómez-Espinosa de los Monteros, R.; Fernández-Santos, J., y Román-Bazán, M. A. (2010). Propuesta para calcular el índice de elasticidad máxima en miembros inferiores. Revista Internacional de Medicina y Ciencias de la Actividad Física y el Deporte, 10(39), 356-368.

Krustrup, P., \& Bangsbo, J. (2001). Physiological demands of top-class soccer refereeing in relation to physical capacity: effect of intense intermittent exercise training. Journal of Sports Science, 19, 881-891.

http://dx.doi.org/10.1080/026404101753113831

Krustrup, P.; Helsen.; W.; Randers, M. B.; Christensen, J. F.; MacDonald, C.; Rebelo, A. N., \& Bangsbo, J. (2009). Activity profile and physical demands of football referees and assistant referees in international games. Journal of Sports Science, 27(11), 1167-1176. http://dx.doi.org/10.1080/02640410903220310

Léger, L., \& Boucher, R. (1980). An indirect continuous running multistage field test: the Université de Montréal track test. Canadian Journal of Applied Sport Science, 5(2), 7784.

Magal, M.; Smith, R. T.; Dyer, J. J., \& Hoffman, J. R. (2009). Seasonal variation in physical performance-related variables in male NCAA Division III soccer players. Journal of Strength and Conditioning Research, 23(9), 2555-2559.

http://dx.doi.org/10.1519/JSC.0b013e3181b3ddbf

Mallo, J.; Navarro, E.; García-Aranda, J. M., \& Helsen, W. (2009). Physical demands of top-class soccer assistant refereeing during high-standard matches. International Journal of Sports Medicine, 30(5), 331-336.

http://dx.doi.org/10.1055/s-0029-1202339

Markovic, G.; Dizdar, D.; Jukic, I., \& Cardinale, M. (2004). Reliability and factorial validity of squat and countermovement jump tests. Journal of Strength and Conditioning Research, 18(3), 551-555.

Maulder, P., \& Cronin, J. (2005). Horizontal and vertical assessment: reliability, symmetry, discriminative and predictive ability. Physical Therapy and Sport, 6, 74-82. http://dx.doi.org/10.1016/j.ptsp.2005.01.001

McGuigan, M. R.; Doyle, T. L.; Newton, M.; Edwards, D. J.; Nimphius, S., \& Newton, R. U. (2006). Eccentric utilization ratio: Effect of sport and phase of training. Journal of Strength and Conditioning Research, 20, 992-995. 
Mendiguchia, J.; Los Arcos, A.; Garrues, M. A.; Myer, G. D.; Yanci, J., \& Idoate, F. (2013). The use of MRI to evaluate posterior thigh muscle activity and damage during nordic hamstring exercise. Journal of Strength and Conditioning Research, 27(12), 34263435.

http://dx.doi.org/10.1519/JSC.0b013e31828fd3e7

Oliveira, R. S.; Leicht, A. S.; Bishop, D.; Barbero-Álvarez, J. C., \& Nakamura, F. Y. (2013). Seasonal changes in physical performance and heart rate variability in high level futsal players. International Journal of Sports Medicine, 34(5), 424-430.

Weston, M.; Castagna, C.; Impellizzeri, F. M.; Rampinini, E., \& Breivik, S. (2010). Ageing and physical match performance in English Premier League soccer referees. Journal of Science and Medicine in Sport, 13(1), 96-100.

http://dx.doi.org/10.1016/j.jsams.2008.07.009

Weston, M.; Drust, B., \& Gregson, W. (2011). Intensities of exercise during match-play in FA Premier League referees and players. Journal of Sports Science, 29(5), 527-532. http://dx.doi.org/10.1080/02640414.2010.543914

Weston, M.; Gregson, W.; Castagna, C.; Breivik, S.; Impellizzeri, F. M., \& Lovell, R. J. (2011). Changes in a top-level soccer referee's training, match activities, and physiology over an 8-year period: a case study. International Journal of Sports Physiology and Performance, 6(2), 281-286.

Weston, M.; Castagna, C.; Impellizzeri, F. M.; Bizzini, M.; Williams, A. M., \& Gregson, W. (2012). Science and medicine applied to soccer refereeing: an update. Sports Medicine, $42,615-631$.

http://dx.doi.org/10.2165/11632360-000000000-00000

Yanci, J., y Los Arcos, A. (2013). Evolución del rendimiento aeróbico y anaeróbico en futbolistas profesionales tras la pretemporada. Cultura, Ciencia y Deporte, 24(8), 207215. 